\section{The DCMS Website - an evaluation}

\section{Juliet Eve}

Juliet Eve is a researcher at Manchester Metropolitan University and a member of the LIRG Committee (with thanks to my colleague Jenny Craven for the information on accessibility issues).

I personally found this rather an irritating site, beginning with the front page, on which the user is confronted with 3 choices of format:

- Standard version of the site

- Animated version, which requires the Flash 4 viewer (link to free download provided)

- Text only version

It is also essential to know what DCMS actually stands for, as the full title of the department is not spelt out.

Choosing the animated version is the fastest route to frustration, as, once a series of unpleasantly coloured lines have raced across the screen, clicking on the links (coloured lines slide off screen to the right) takes you to a blank white screen. Having tried 3 links, only to have to press the 'Back' button and return to the start page, I gave up on the dubious delights of the animated version.

The standard version takes you to the page of unpleasant stripes, but at least they don't fly across the page making you feel sick. From here, you can choose one of eight links, including the link to the text only version. Each link takes you to a page (different colour shemes for each, none of which make for easy browsing or reading; particularly difficult is the pink 'creative, media \& arts' page) containing small snippets of news, some 'facts and figures' and a choice of 3-5 main headings for further information. The 'heritage, libraries \& museums' link for example, contains further links to: museums \& galleries, libraries and historic environment. The website does not exactly overwhelm the user with information; for instance, one page is devoted to public libraries (in orange and brown); this has a bit of blurb about the role of public libraries, and 7 links, including one to the site which hosts Annual Library Plans. I imagine that useful information is there, but it is incredibly hard to locate it. To find specific information on the People's Network, you have to search for it, (and there is no search button on the main page) though the DCMS/Wolfson Fund and the "The Public Libraries ICT Network" are briefly mentioned under 'education and ICT' on another page. There is also no 'Home' button on any of the pages, and it took a while to discover that the small squares at the top of each page are well-disguised links to the other sections, as this is only revealed if you move the mouse over them.

\section{Accessibility}

So much for the content, what about accessing it? Leaving aside the animated version, how do the standard and text-only stand up to scrutiny? The standard site uses frames, so will not be approved by Bobby, a popular accessibility checker. The font size is quite small and not easy to read (even for those without visual difficulties), and colours chosen for pages are not always contrasting (e.g. two shades of aqua for background and text). Graphics are used with no text alternative - perhaps assuming that people using assistive technology will go straight to the text only version. The text only version is, however, very accessible (though somewhat uninteresting) apart from some of the links at the bottom of the page which could be better spaced. All in all, it seems highly unnecessary to provide three versions of one site, when it is possible to provide one that is interesting, yet accessible.

Designers seem to have tried to create a flashy, innovative site rather at the cost of accessibility. It is all very New Labour infact, the triumph of form over substance.......

(site accessed 5 April 2000 \& 26 May 2000)

DCMS stands for Department for Culture, Media \& Sport and is at www.culture,gov.uk. (Department name now appears - ED.) 